\title{
Expression of focal adhesion kinase in the eutopic endometrium of women with adenomyosis varies with dysmenorrhea and pelvic pain
}

\author{
LIN MU, WEIMIN CHEN, YANYAN MA and WEI ZHENG \\ Department of Gynecology, The Second Affiliated Hospital, School of Medicine, \\ Zhejiang University, Hangzhou, Zhejiang 310009, P.R. China \\ Received November 3, 2014; Accepted July 31, 2015
}

DOI: $10.3892 /$ etm.2015.2736

\begin{abstract}
The aim of the present study was to examine whether the expression of focal adhesion kinase (FAK) is altered in the eutopic endometrium of female patients with adenomyosis, as compared with that of females without adenomyosis. The expression of FAK was assessed by immunohistochemical, western blot and reverse transcription-quantitative polymerase chain reaction analyses. An elevated expression of FAK mRNA and protein was identified in the eutopic endometrium of patients with adenomyosis compared with patients without adenomyosis $(\mathrm{P}<0.05)$. In addition, a positive correlation was detected between FAK protein expression and dysmenorrhea and pelvic pain in females with adenomyosis $(\mathrm{P}<0.05)$. The significant increase of FAK expression identified in the eutopic endometrium of females with adenomyosis, as well as the association of FAK protein expression with dysmenorrhea and pelvic pain, suggested that FAK may play a role in the pathogenesis of adenomyosis.
\end{abstract}

\section{Introduction}

Adenomyosis, one of the most common debilitating diseases, affects women in the reproductive age group (1). Uterine adenomyosis, by definition, is the presence of endometrial tissue, including stroma and glands, $\geq 2.5 \mathrm{~mm}$ below the endometrial-myometrial junction and widely distributed within the myometrium layer of the uterus. An adenomyoma is a circumscribed, nodular aggregate of smooth muscle, and endometrial glands and stroma within the myometrium (2). Common clinical symptoms of adenomyosis include metrorrhagia, dysmenorrhea, pelvic pain, early pregnancy-stage

Correspondence to: Dr Wei Zheng, Department of Gynecology, The Second Affiliated Hospital, School of Medicine, Zhejiang University, 88 Jiefang Road, Hangzhou, Zhejiang 310009, P.R. China E-mail: zhengwei@zju.edu.cn

Key words: adenomyosis, focal adhesion kinase, eutopic endometrium, dysmenorrhea, pelvic pain miscarriage, menorrhagia and subfertility (3). The management of adenomyosis has been a major challenge, with hysterectomy comprising the treatment of choice (4). The ontogeny of adenomyosis is clearly important for the development of new alternatives to hysterectomy. Despite the frequency of the disease, its precise etiology and physiopathology remain unknown. The current theory is that the disease is developed through the downgrowth and invagination of the basalis endometrium into the myometrium (5). According to the aforementioned theory, the increased invasiveness of the endometrial cells may result in the development of adenomyosis.

Several studies have reported that estrogen-induced epithelial-to-mesenchymal transition (EMT) is critical to the pathogenesis of adenomyosis (6,7). EMT is an important developmental program exploited by cancer cells in their acquisition of invasive and metastatic capacity (8). Its distinctive characteristics are the loss of E-cadherin and apical-basal cell polarity, accompanied by the acquisition of cell migration and invasion abilities and an increased expression of mesenchymal markers, including fibronectin, $\mathrm{N}$-cadherin and vimentin (9). A number of recent studies have implicated focal adhesion kinase (FAK) in the regulation of EMT (10-12). FAK was shown to mediate cell invasion and metastasis through the promotion of EMT; however, whether FAK is involved in the pathology of adenomyosis has yet not been explored.

The aims of the present study were therefore to investigate whether there was a difference in FAK expression in the eutopic endometria of women with and without adenomyosis, and to examine whether FAK expression in adenomyosis of the eutopic endometrium was associated with pelvic pain and dysmenorrhea.

\section{Materials and methods}

Patients and sample collection. The ethical approval for the present study was obtained by the Ethics Committee of School of Medicine, Zhejiang University (Hangzhou, China). Written informed consent was obtained from all the patients prior to tissue collection. A total of 47 females in reproductive age volunteered to participate in the present study. All the participants had normal menstrual cycles (28-32 days) and had 
not received any anti-inflammatory or hormonal treatment for $\geq 6$ months prior to enrollment and surgery.

Of the 47 patients, 22 females (aged 39-45 years) had been diagnosed with adenomyosis by laparoscopy and subsequent histological analysis. Information on the history of pelvic pain and dysmenorrhea was obtained from the patients' clinical records. The standard visual analogue scale was used to measure pain intensity (13). The patients were taught to rate their pain intensity in a scale of 1-10. The control group comprised 25 females (aged 38-43 years), who were undergoing hysterectomy for benign indications and had no visible evidence of adenomyosis or endometriosis. The mean ages of the patients with adenomyosis and the control group were $41.7 \pm 3.8$ years and $40.9 \pm 2.9$ years, respectively, and no statistically significant age difference was observed between them $(\mathrm{P}>0.05)$.

Endometrial tissues were obtained by endometrial curettage (Pipelle, Laboratoire CDD, Paris, France) simultaneously with the surgery. Shortly after the tissue collection, the endometrial tissues were either snap-frozen in liquid nitrogen and stored at $-80^{\circ} \mathrm{C}$ for mRNA and protein extraction, or fixed for $24 \mathrm{~h}$ in $4 \%$ paraformaldehyde for pathological examination and embedded in paraffin for immunohistochemical analysis.

Immunohistochemistry. The endometrial samples were sectioned at $4-\mu \mathrm{M}$ intervals, and the slides were heated at $60^{\circ} \mathrm{C}$ for $1 \mathrm{~h}$, deparaffinized in xylene and washed with graded ethanol solutions followed by distilled water. Next, the slides were heated to $92-98^{\circ} \mathrm{C}$ in $0.01 \mathrm{~mol} / 1$ sodium citrate buffer for $15 \mathrm{~min}$ and then placed at room temperature for $30 \mathrm{~min}$. Subsequently, the slides were incubated with $3 \%$ (v/v) hydrogen peroxide for $10 \mathrm{~min}$. Non-specific binding was blocked by $10 \%$ (v/v) normal goat serum in PBS for 10 min at room temperature, and the slides were incubated with the anti-FAK primary antibody (dilution, 1:100) in PBS for $2 \mathrm{~h}$ at room temperature.

The anti-FAK antibody used in the present study was a rabbit polyclonal antibody of mouse origin, raised against amino acids 903-1052 of FAK (cat. no. sc-932; Santa Cruz Biotechnology, Inc., Santa Cruz, CA, USA). Slides incubated with a rabbit immunoglobulin ( $\mathrm{IgG}$ ) antibody at the same dilution as the primary antibody were used as negative controls. Following three washes with PBS for 5 min each, slides were incubated with anti-IgG horseradish peroxidase (HRP)-conjugated secondary antibody (cat. no. P0488; Dako Cytomation, Inc., Carpinteria, CA, USA) for $30 \mathrm{~min}$. After a further wash, the sections were treated with diaminobenzidine (Dako Cytomation, Inc.), counterstained with hematoxylin (Sigma-Aldrich, St. Louis, MO, USA), dehydrated and mounted in DPX mounting medium (Merck Millipore, Darmstadt, Germany).

Western blotting. The homogenization of the endometrial samples was performed in $1 \mathrm{X}$ radioimmunoprecipitation assay buffer (Sigma-Aldrich) that contained 1\% Nonidet P-40, $0.1 \%$ SDS, $0.05 \%$ deoxycholate and protease inhibitors $(1 \mu \mathrm{g} / \mathrm{ml}$ phenylmethylsulfonyl fluoride and $1 \mu \mathrm{g} / \mathrm{ml}$ leupeptin). The homogenate was incubated for $40 \mathrm{~min}$ on ice and subsequently centrifuged at $15,000 \mathrm{x} \mathrm{g}$ for $5 \mathrm{~min}$ at $4^{\circ} \mathrm{C}$. The insoluble fraction was discarded. A Bradford assay (Bio-Rad Laboratories, Inc., Hercules, CA, USA) was used to determine the protein concentration in the supernate and then $50 \mu \mathrm{g}$ proteins were added into $2 \mathrm{X}$ sodium dodecyl sulfate (SDS) buffer and heated for $5 \mathrm{~min}$ at $95^{\circ} \mathrm{C}$. Samples at $50 \mu \mathrm{g} /$ lane were separated on a $8 \%$ SDS-polyacrylamide gel and then transferred to an immune-blot nitrocellulose transfer membrane (Protran ${ }^{\circledR}$; Schleicher \& Schuell BioScience GmbH, Dassel, Germany).

The membranes were blocked with $5 \%$ non-fat milk powder in Tris-buffered saline/Tween-20 (TBST), and then incubated with the anti-FAK (dilution, 1:200) and $\beta$-actin (dilution, 1:400; sc-47778; Santa Cruz Biotechnology, Inc.) primary antibodies at $4^{\circ} \mathrm{C}$ overnight. Following several washes with TBST, incubation of the membranes was performed for $2 \mathrm{~h}$ at room temperature with the secondary HRP-labeled antibody (dilution, 1:5,000). The bound antibody was detected using an enhanced chemiluminescence detection reagent (Santa Cruz Biotechnology, Inc.). The bands were scanned using Quantity One software, version 4.62 (Bio-Rad Laboratories, Inc.). Normalized densities were determined using the ratio of the band density of FAK to the band density of $\beta$-actin.

Reverse transcription-quantitative polymerase chain reaction (RT-qPCR) analysis. In order to validate the results of the array, semi-quantitative RT-qPCR was used to examine the FAK mRNA expression. Total RNA was extracted from each endometrial sample using TRIzol reagent (Invitrogen Life Technologies, Carlsbad, CA, USA). Reverse transcription was performed with $3 \mu \mathrm{g}$ RNA using random primers and M-MLV reverse transcriptase (Promega Corporation, Madison, WI, USA) according to the manufacturer's instructions. The PCR reaction for FAK was performed in a final volume of $25 \mu 1$, using 0.25 units Taq DNA polymerase (Sangon Biotech Co., Ltd., Shanghai, China), $0.2 \mu \mathrm{M}$ of each primer and $200 \mu \mathrm{M}$ deoxynucleotide triphosphate in $10 \mathrm{nM}$ Tris- $\mathrm{HCl}$ buffer $(\mathrm{pH} 8.3)$, containing $50 \mathrm{nM} \mathrm{KCl}$ and $1.5 \mathrm{nM} \mathrm{MgCl}_{2}$. GADPH was used as the endo-reference control. The FAK primer sequences used were as follows: Sense, 5'-AATACGGCGATCATACTGGG-3', and anti-sense, 5'-CAT GCCTTGCTTTTCGCTGT-3', amplifying a 620 bp product; GAPDH sense, 5'-ACCACAGTCCATGCCATCAC-3', and anti-sense, 5'-TCCACCACCCTGTTGCTGTA-3', amplifying a 452 bp product. PCR was performed in a DNA thermal cycler using the following conditions: 1 cycle of $95^{\circ} \mathrm{C}$ for $5 \mathrm{~min}$, then 40 cycles of $95^{\circ} \mathrm{C}$ for $30 \mathrm{sec}, 54^{\circ} \mathrm{C}$ for $30 \mathrm{sec}$ and $72^{\circ} \mathrm{C}$ for $30 \mathrm{sec}$, and finally 1 cycle of $72^{\circ} \mathrm{C}$ for $10 \mathrm{~min}$. Subsequently, $10 \mu \mathrm{lPCR}$ product mixed with $2 \mu 1$ loading buffer were electrophoretically separated on a $2 \%$ agarose gel and visualized with ethidium bromide (Life Technologies, Carlsbad, CA, USA).

Statistical analysis. All data were normally distributed and the results are expressed as the mean \pm standard deviation. Statistical analysis of the FAK to endo-reference ratios was performed by one-way analysis of variance using SPSS software, version 11.5 (SPSS, Inc., Chicago, IL, USA). Linear regression was used to analyze the correlation between FAK protein expression and the score of pelvic pain and dysmenorrhea. $\mathrm{P}<0.05$ was considered to indicate a statistically significant difference.

\section{Results}

Immunohistochemistry. FAK immunoreactive staining was present in the cytoplasm of glandular epithelial and stromal 
A

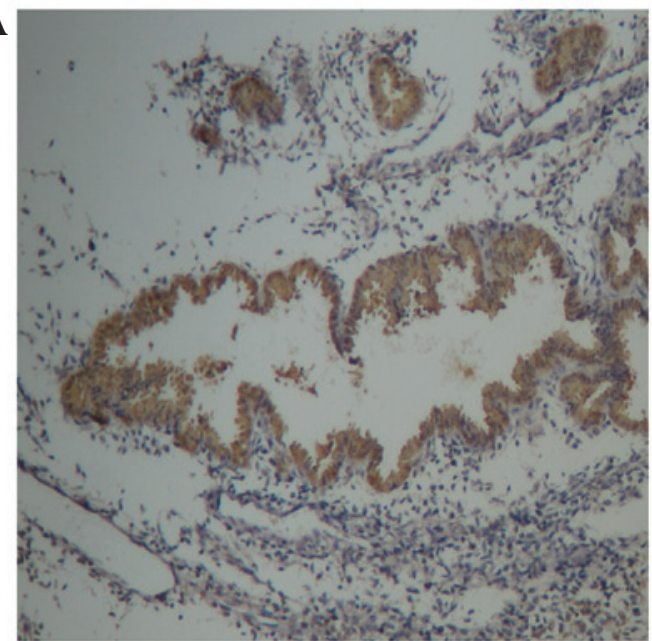

B

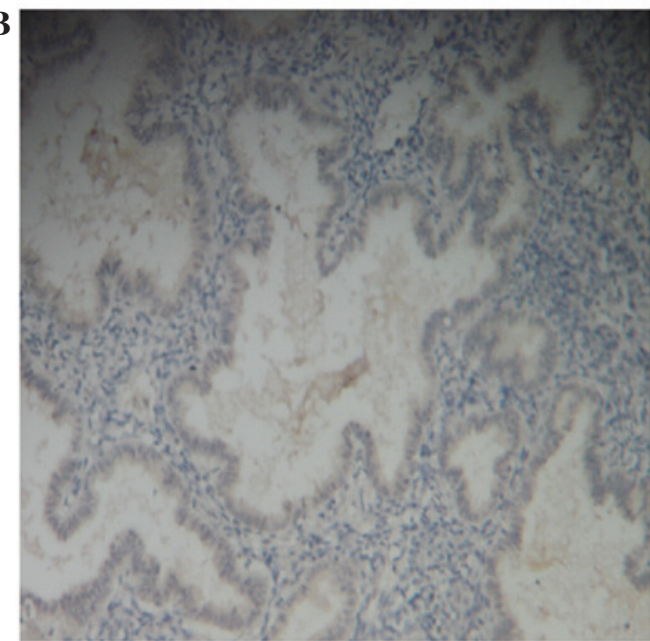

Figure 1. Immunohistochemical staining for focal adhesion kinase in the endometrium of females (A) with adenomyosis and (B) without adenomyosis. Original magnification, $\mathrm{x} 200$.

A

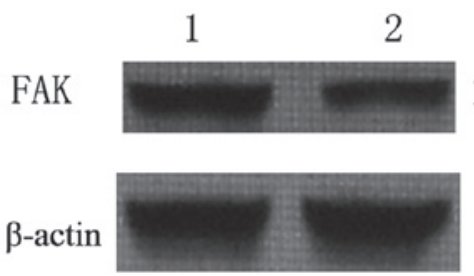

$125 \mathrm{kDa}$

$43 \mathrm{kDa}$

B

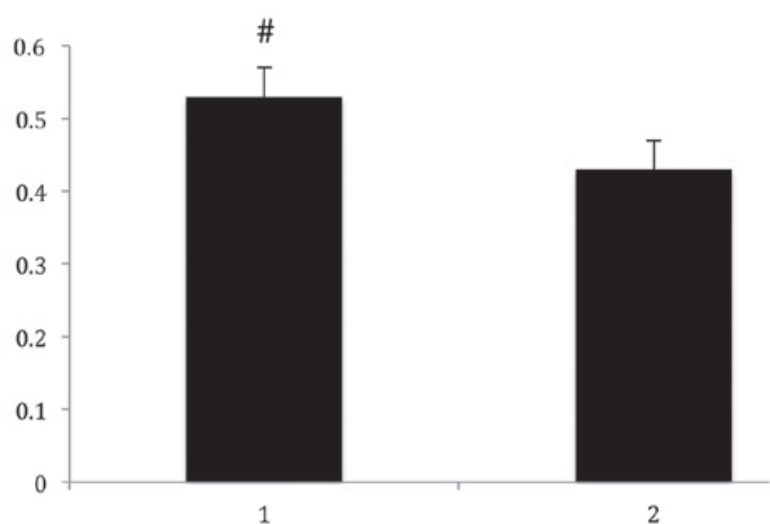

Figure 2. (A) FAK protein expression in endometrial tissues was determined by western blotting. The anti-FAK antibody detected a band at $125 \mathrm{kDa}$ (B) Normalized density was analyzed using the internal $\beta$-actin as a reference (mean \pm standard deviation. ${ }^{\sharp} \mathrm{P}=0.020$, vs. control patients. 1 , eutopic endometrium from female patients with adenomyosis; 2, eutopic endometrium from females without adenomyosis; FAK, focal adhesion kinase.

cells in females with and without adenomyosis (Fig. 1A and B, respectively). The immunostaining in the glandular epithelium was more evident.

Western blot analysis. The anti-FAK antibody detected a band at $125 \mathrm{kDa}$ in protein extracts from all the endometrial tissues (Fig. 2A). Following the normalization of each band of FAK with $\beta$-actin from different samples, the average FAK expression in patients with adenomyosis $(0.53 \pm 0.04)$ was found to be significantly higher compared with that of the control group $(0.43 \pm 0.05 ; \mathrm{P}<0.05$; Fig. $2 \mathrm{~B})$.
A

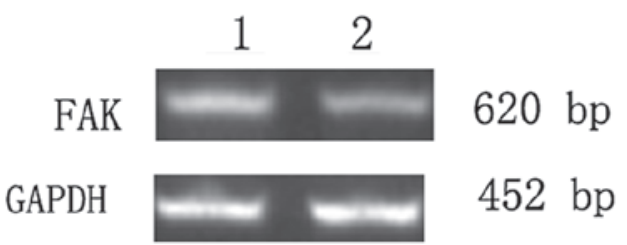

B

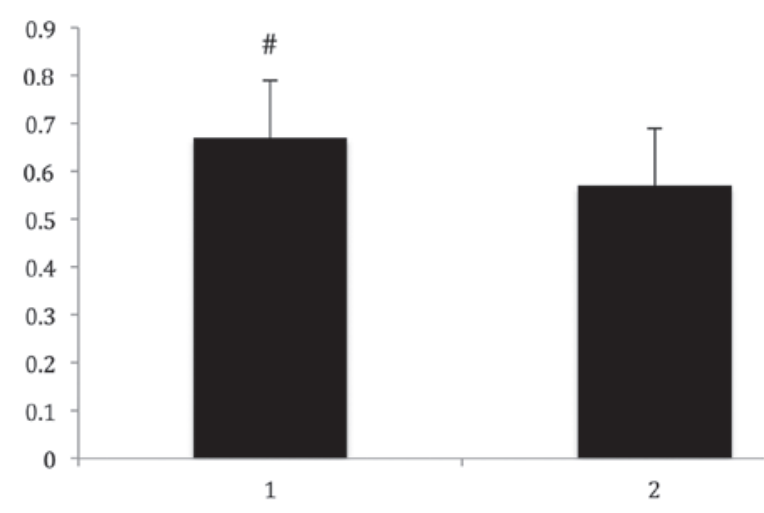

Figure 3. (A) FAK mRNA expression was determined by reverse transcription-quantitative polymerase chain reaction in endometrial samples. A 620 bp product of FAK mRNA was visualized with ethidium bromide after agarose gel electrophoresis. GAPDH (452 bp) was used as a control to assess the amount of RNA in each sample. (B) Quantification of FAK mRNA expression was analyzed using the internal GAPDH as a reference (expressed as the mean \pm standard deviation). ${ }^{\#} \mathrm{P}=0.030$, vs. control patients. 1 , eutopic endometrium from female patients with adenomyosis; 2, eutopic endometrium from females without adenomyosis; FAK, focal adhesion kinase.

RT-qPCR analysis. RT-qPCR was used to assess the FAK mRNA expression, and a 620-bp product of FAK mRNA was visualized with ethidium bromide following agarose gel electrophoresis. GADPH was used as a control to assess the volume of RNA in each sample (Fig. 3A).

Semi-quantitative PCR analysis identified that FAK mRNA expression in the endometrial samples of patients with adenomyosis was significantly higher $(0.67 \pm 0.12)$ compared with that of the control individuals $(0.57 \pm 0.11 ; \mathrm{P}<0.05$; Fig. 3B). 


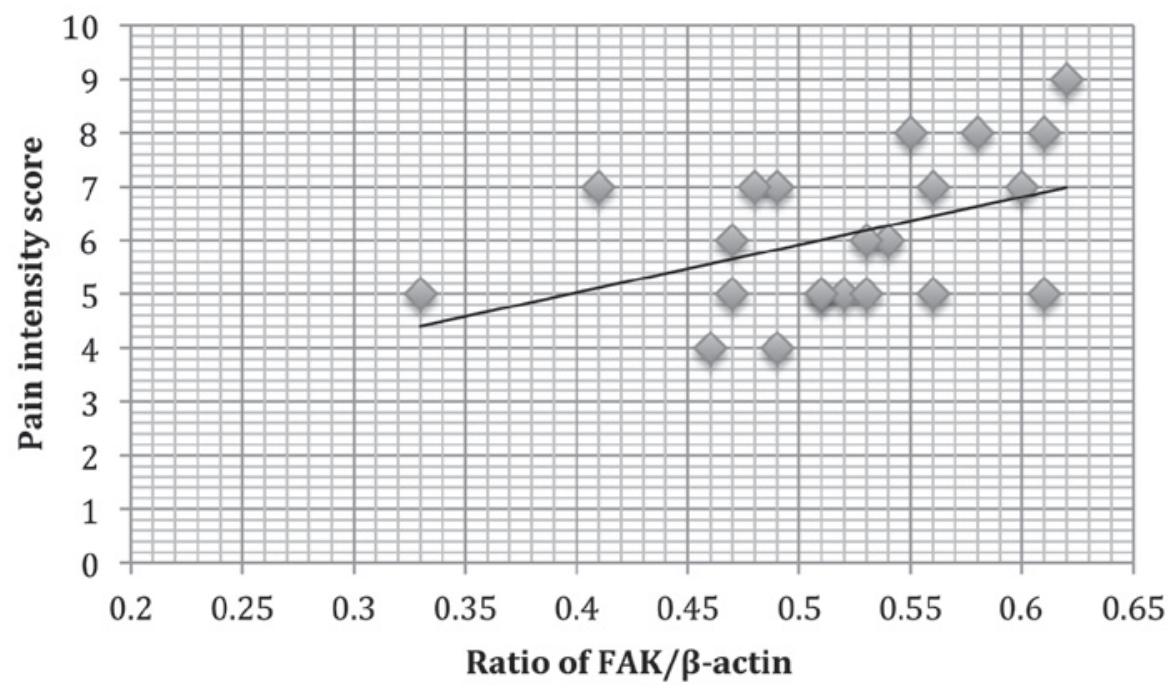

Figure 4. A positive correlation was observed between the normalized densities of endometrial FAK proteins and the pain intensity score of dysmenorrhea $\left(\mathrm{n}=22 ; \mathrm{r}^{2}=0.121, \mathrm{P}=0.011\right)$. FAK, focal adhesion kinase.

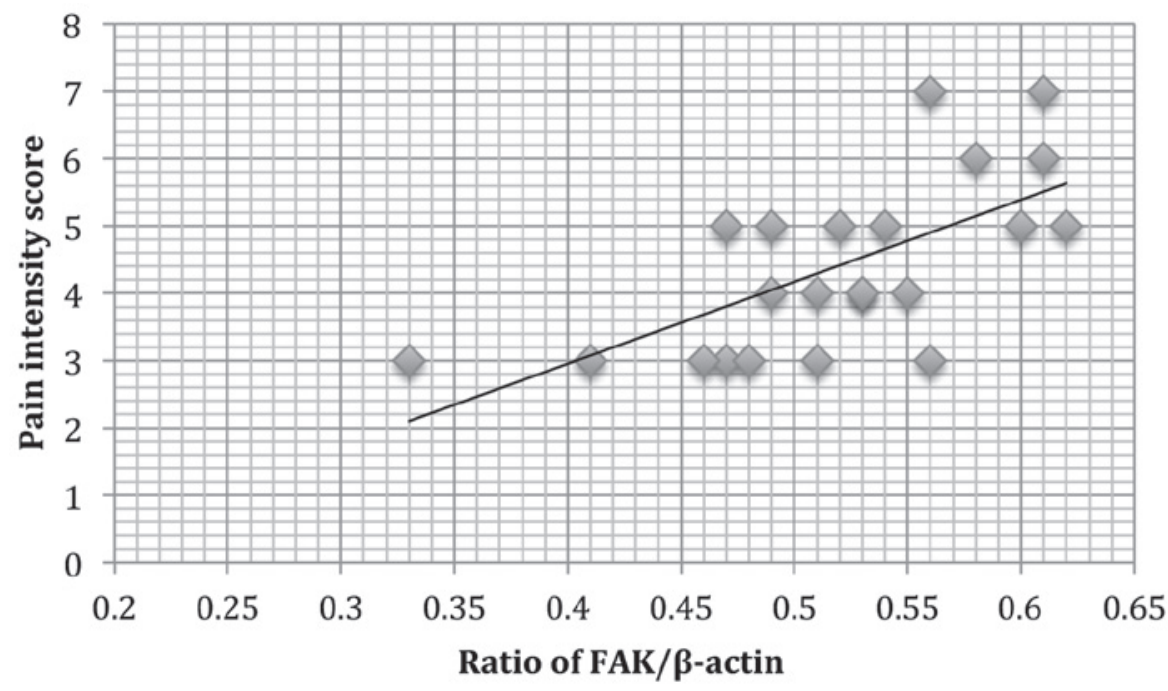

Figure 5. A positive correlation was observed between the normalized densities of endometrial FAK proteins and the pain intensity score of pelvic pain ( $\mathrm{n}=22$; $\left.\mathrm{r}^{2}=0.110, \mathrm{P}=0.009\right)$. FAK, focal adhesion kinase.

Correlation of FAK protein expression with dysmenorrhea and pelvic pain in adenomyosis. A positive correlation was observed between FAK protein expression and dysmenorrhea $\left(r^{2}=0.121, P=0.011\right.$; Fig. 4$)$ in patients with adenomyosis. In addition, a positive correlation of pelvic pain and FAK protein expression $\left(r^{2}=0.110, \mathrm{P}=0.009\right.$; Fig. 5) was observed.

\section{Discussion}

In the present study, an elevation in FAK expression was observed in the eutopic endometrium of patients with adenomyosis. In addition, an association was identified between FAK protein expression in endometrial tissues from patients with adenomyosis and the scores of dysmenorrhea and pelvic pain. These results provide further evidence that the eutopic endometrium of patients with adenomyosis is aberrant, and the downgrowth and invagination of the basalis endometrium into the myometrium is critical to the pathogenesis of adenomyosis.
FAK is a part of the integrin-stimulated signaling network and is localized to sites of integrin clustering at focal adhesions through indirect protein-protein interactions (14). The N-terminus is a FERM domain reported to be in direct association with the cytoplasmic tail of integrins (15). The focal adhesion targeting sequence that mediates discrete localization to focal adhesions is known to reside in the C-terminal non-catalytic domain of FAK. The remaining region in this domain is more adjacent to the catalytic domain and consists of two proline-rich sequences functioning as binding sites for a variety of Src (16), which may result in the activation of multiple intracellular signaling cascades. Through the aforementioned binding sites, FAK controls several biological processes, such as cell survival, proliferation, invasion and migration (17). FAK is overexpressed in several types of solid and non-solid tumors, mediating survival and other important functions (18). Due to the fact that high percentages of FAK overexpression have been reported in different types of tumors, FAK has been proposed as a therapeutic target (19). 
In addition to determining the role of FAK in cell proliferation, survival and migration, recent studies have also revealed potentially novel functions of FAK in the regulation of EMT (20), an important developmental program exploited by cancer cells in their process of acquiring invasive and metastatic capacity. Chen et al have suggested that oestrogen-induced EMT of endometrial epithelial cells contributes to the development of adenomyosis (7). According to the results of the present study, we postulate that FAK may be involved in the EMT of adenomyosis. The connection between the loss of E-cadherin expression by cancer cells and passage through an EMT has been established by numerous studies $(21,22)$. FAK was shown to affect E-cadherin expression through different mechanisms. The phosphorylation of FAK was required for the Src-induced downregulation of E-cadherin in colon cancer cells (23). Thus, we speculate that the higher expression of FAK in adenomyosis caused the deregulation of E-cadherin, promoting EMT and the invasion and metastasis of endometrial cells. Based on this speculation, it is very likely that FAK plays a critical role in transforming the eutopic endometrium of adenomyosis in order to be more susceptible to surviving, adhering and growing in the ectopic sites.

In conclusion, to the best of our knowledge, the present study revealed for the first time a significant increase in FAK expression in the endometrial tissues of female patients with adenomyosis, as well as an association of FAK expression with dysmenorrhea and pelvic pain. These findings suggest that FAK may contribute to the pathogenesis of adenomyosis. Further research is warranted on the application of FAK as a clinical marker and therapeutic target.

\section{Acknowledgements}

This study was supported by a grant from the National Natural Science Foundation of China (grant no. 81100407).

\section{References}

1. Vercellini P, Vigano P, Somigliana E, Daguati R, Abbiati A and Fedele L: Adenomyosis: Epidemiological factors. Best Pract Res Clin Obstet Gynaecol 20: 465-477, 2006.

2. Bergeron C, Amant F and Ferenczy A: Pathology and physiopathology of adenomyosis. Best Pract Res Clin Obstet Gynaecol 20: 511-521, 2006.

3. Peric $\mathrm{H}$ and Fraser IS: The symptomatology of adenomyosis. Best Pract Res Clin Obstet Gynaecol 20: 547-555, 2006.

4. Farquhar $\mathrm{C}$ and Brosens I: Medical and surgical management of adenomyosis. Best Pract Res Clin Obstet Gynaecol 20: 603-616, 2006.
5. Parrott E, Butterworth $\mathrm{M}$ and Green A: Adenomyosis-a result of disordered stromal differentiation. Am J Pathol 159: 623-630,2001.

6. Oh SJ, Shin JH and Kim TH: $\beta$-Catenin activation contributes to the pathogenesis of adenomyosis through epithelial-mesenchymal transition. J Pathol 231: 210-222, 2013.

7. Chen YJ, Li HY and Huang CH: Oestrogen-induced epithelial-mesenchymal transition of endometrial epithelial cells contributes to the development of adenomyosis. J Pathol 222: 261-270, 2010.

8. Acloque H, Adams MS and Fishwick K: Epithelial - mesenchymal transitions: The importance of changing cell state in development and disease. J Clin Invest 119: 1438-1449, 2009.

9. Polyak K and Weinberg RA: Transitions between epithelial and mes- enchymal states: Acquisition of malignant and stem cell traits. Nat Rev Cancer 9: 265-273, 2009.

10. Scheel C and Weinberg RA: Phenotypic plasticity and epithelial-mesenchymal transitions in cancer and normal stem cells? Int J Cancer 129: 2310-2314, 2011.

11. Fan H, Zhao X, Sun S, Luo M and Guan JL: Focal adhesion kinase scaffolding function to mediate endophilin A2 phosphorylation promotes epithelial-mesenchymal transition and mammary cancer stem cell activities in vivo. J Biol Chem 288: 3322-3333, 2013.

12. Serrels A, Canel M, Brunton VG and Frame MC: Src/FAK-mediated regulation of E-cadherin as a mechanism for controlling collective cell movement: Insights from in vivo imaging. Cell Adh Migr 5: 360-365,2011.

13. Knop C, Oeser M, Bastian L, Lange U, Zdichavsky M and Blauth M: Development and validation of the Visual Analogue Scale (VAS) Spine Score. Unfallchirurg 104: 488-497, 2001 (In German).

14. Cox BD, Natarajan M, Stettner MR and Gladson CL: New concepts regarding focal adhesion kinase: Promotion of cell migration and proliferation. J Cell Biochem 99: 35-52, 2006.

15. Schaller MD: Biochemical signals and biological responses elicited by the focal adhesion kinase. Biochim Biophys Acta 1540: 1-21, 2001

16. Schaller MD, Hildebrand JD and Shannon JD: Autophosphorylation of the focal adhesion kinase, pp125FAK, directs SH2-dependent binding of pp60src. Mol Cell Biol 14: 1680-1688, 1994

17. Zhang X, Chattopadhyay A and Ji QS: Focal adhesion kinase promotes phospholipase C-gammal activity. Proc Natl Acad Sci USA 96: 9021-9026, 1999.

18. Gabarra-Niecko V, Schaller MD and Dunty JM: FAK regulates biological processes important for the pathogenesis of cancer. Cancer Metastasis Rev 22: 359-374, 2003.

19. McLean GW, Avizienyte E and Frame MC: Focal adhesion kinase as a potential target in oncology. Expert Opin Pharmacother 4: 227-234, 2003.

20. Canel M, Serrels A, Frame MC and Brunton VG: E-cadherin-integrin crosstalk in cancer invasion and metastasis. J Cell Sci 126: 393-401, 2013.

21. Tepass U, Truong K, Godt D. Ikura M and Peifer M: Cadherins in embryonic and neural morphogenesis. Nat Rev Mol Cell Biol 1: 91-100, 2000

22. Edelman GM, Gallin WJ, and Delouvee A: Early epochal maps of two different cell adhesion molecules. Proc Natl Acad Sci 80: 4384-8, 1983

23. Avizienyte E, Wyke AW and Jones RJ: Src-inducd de-regulation of E-cadherin in colon cancer cells requires integrin signalling. Nat Cell Biol 4: 632-638, 2002. 